\title{
Measuring motor speed through typing: a surrogate for the finger tapping test
}

\author{
Daniel Austin • Holly Jimison • Tamara Hayes • \\ Nora Mattek • Jeffrey Kaye • Misha Pavel
}

Published online: 15 April 2011

(C) Psychonomic Society, Inc. 2011

\begin{abstract}
Motor speed is an important indicator and predictor of both cognitive and physical function. One common assessment of motor speed is the finger-tapping test (FTT), which is typically administered as part of a neurological or neuropsychological assessment. However, the FTT suffers from several limitations, including infrequent in-person administration, the need for a trained assessor and dedicated equipment, and potential shortterm sensory-motor fatigue. In this article, we propose an alternative method of measuring motor speed, with face validity to the FTT, that addresses these limitations by measuring the interkeystroke intervals (IKI) of familiar and repeated login data collected in the home during a subject's regular computer use. We show significant correlations between the mean tapping speeds from the FTT and the median IKIs of the nondominant $(r=.77)$ and dominant $(r=.70)$ hands, respectively, in an elderly cohort of subjects living independently. Finally, we discuss how the proposed method
\end{abstract}

D. Austin $(\bowtie) \cdot$ T. Hayes $\cdot$ M. Pavel

Department of Biomedical Engineering,

Oregon Health and Science University,

3303 SW Bond Avenue MC: CH13B,

Portland, OR 97239, USA

e-mail: austidan@bme.ogi.edu

\section{H. Jimison}

Department of Medical Informatics and Clinical Epidemiology,

Oregon Health and Science University,

3181 SW Sam Jackson Park Rd. MC: BICC,

Portland, OR 97239, USA

N. Mattek $\cdot$ J. Kaye

Layton Aging and Alzheimer's Disease Center,

Oregon Health and Science University,

3181 SW Sam Jackson Park Rd. MC: CR131,

Portland, OR 97239, USA for measuring motor speed fits well into the framework of unobtrusive and continuous in-home assessment.

Keywords Motor speed - Typing · Finger tapping test

Sensory-motor speed is an important predictor of cognitive and physical functionality, which are some of the key determinants of individual well being. For example, motor slowing, as indicated by finger-tapping speed and walking speed measurements, have been shown to precede cognitive impairment in the elderly (Camicioli, Howieson, Oken, Sexton, \& Kaye, 1998), and slow motor speed has been shown to be a risk factor for fractures during falls (Shigematsu, Rantanen, Saari, Sakari-Rantala, Kauppinen, Sipila et al., 2006). One common assessment of motor speed is the finger-tapping test (FTT). The FTT is frequently used as part of a neuropsychological examination to detect both motor and cognitive impairments (Strauss, Sherman, \& Spreen, 2006). This test is typically scored as the average number of times a patient can depress a key with his or her index finger (each hand is tested separately) on a manual finger-tapping device in $10 \mathrm{~s}$. The test nominally consists of 5 tapping trials, subject to the constraints that either the counts on all trials are within five of each other or no more than 10 trials are administered (Strauss et al., 2006). Under this procedure, extra trials are administered only if the first condition is not met, although there are many other variants described in the literature.

The FTT and similar tests have been used for assessment of movement slowing related to aging in general (Godefroy, Roussel, Despretz, Quaglino, \& Boucart, 2010; JiménezJiménez et al., 2011; Kaye, Oken, Howieson, Howieson, Holm, \& Dennison, 1994) as well as to a number of medical conditions, including stroke (Godefroy, Spagnolo, 
Roussel, \& Boucart, 2010), essential tremor (JimenezJimenez, Rubio, Alonso-Navarro, Calleja, Pilo-de-la-Fuente, Plaza-Nieto et al., 2010), and Alzheimer's disease (Ott, Ellias, \& Lannon, 1995; Wefel, Hoyt, \& Massma, 1999). This type of testing may be especially useful in high-risk patient populations, such as patients with mild cognitive impairment, who are at greater risk to convert to Alzheimer's disease (Buracchio, Dodge, Howieson, Wasserman, \& Kaye, 2010; Levey, Lah, Goldstein, Steenland, \& Bliwise, 2006), or the elderly, who are at increased risk for many adverse outcomes.

Despite the utility and successful application of the FTT, the test still suffers from several shortcomings. First, because it is performed with an in-person assessor, the test is usually administered infrequently - typically no more often than once every 6 months, and frequently with intervals of a year or more between assessments. Second, a trained assessor is required to administer the standard test using a stopwatch and a manual finger tapper; using an alternative, computerized tapping test apparatus still requires an assessor to instruct the test volunteer. As a result of the first limitation, the FTT cannot reliably detect motor changes at the time of onset or distinguish between acute changes and slower changes that have occurred over time. Furthermore, when a change is detected between two visits, it is difficult to determine whether this has been caused by inherent test performance variability between the two examination dates or whether there has been a true change in motor function. The requirement for an in-person test administrator on repeated examinations adds to the expense of administering the FTT and also introduces concerns with interrater reliability (Morrison, Gregory, \& Paul, 1979). There has been some attempt to standardize the FTT into a computer-based test (Christianson \& Leathem, 2004; Kiziltan, Barut, \& Gelir, 2006) that would solve the interrater issues, but the computer-based methods still require trained personnel to give the test and do not solve the issue of infrequent measurements. In addition to these shortcomings, the results of the FTT confound motor ability with short-term fatigue, which is especially noticeable after several trials. Finally, the FTT itself is not a natural task - that is, we do not normally perform tapping movements - which limits its everyday or ecological validity.

In this article, we propose a surrogate for the FTT based on monitoring the interkeystroke interval (IKI) of repeated computer login data, and we validate this measure in a group of 22 elderly subjects who live alone and are monitored remotely and unobtrusively in their homes. Specifically, we compare the relationship between the average tapping speeds calculated from FTT scores (denoted as $T_{\mathrm{FTT}}$ ) of both the dominant and nondominant hands with the relationship between the median IKI from keystrokes executed while entering overlearned and familiar sequences, such as the user name string typed during each computer login. Prior research suggested that typing familiar words - for example, words with a high frequency of occurrence - is much faster than typing low-frequency words or random letters (Gentner, Larochelle, \& Grudin, 1988; Shaffer, 1973). By minimizing the cognitive load and keyboard search time during the execution of these overlearned sequences, we hypothesized that median IKIs could be a potentially valid measure of raw motor speed. The action of typing also has face validity, in that the flexion and extension of the finger required to depress and release a key is similar to that required to depress and release the lever on the manual finger-tapper board. Additionally, with more and more people owning personal computers and laptops, collecting IKI data can be as simple as installing a software key logger on a patient's personal computer with a known login sequence or providing a patient with a computer that has software already installed. This new approach offers a low-cost measure of motor speed with reduced subject burden. In addition, repeated measures in a natural environment allow us to measure within-subjects trends over time and potentially to detect problems much earlier than traditional methods. This detailed tracking may facilitate continuous assessment of high-risk patients and frequent and widespread assessments of subjects in clinical trials with reduced cost and objective measurements.

\section{Method}

\section{Subjects}

Our subject pool consisted of the computer arm of the Intelligent Systems for Assessing Aging Changes (ISAAC) cohort of 225 elders living in homes and retirement communities in the Portland, Oregon (USA), metropolitan area. The overall ISAAC study and cohort are described in more detail elsewhere (Kaye et al., in press); our description here will consist only of the details related to computer use and the data inclusion criteria for the subjects used in the present analysis. Subjects who lived alone and who used their computers frequently during a 28-day window centered on the date of their in-person-administered FTT assessment were considered for inclusion. To ensure that sufficient keystroke data were available for analysis, we further required that subjects had entered at least 80 characters of user name data during the 28-day window centered on the associated FTT date. This corresponds roughly to the requirement that the subject $\log$ in to the computer on at least half of the days in the data window (mean user name length was 5.7 characters; 80/5.7 is approximately 14 logins). These criteria ensured that the 
key capture data obtained from the subject's login belonged to the subject and that there were sufficient data to calculate a reasonable measure of the central tendency of IKIs near each FTT date for comparison.

One final exclusionary criterion was used as a method for outlier identification, based on the residual error from a linear regression (described in more detail below). We identified as outliers those data points whose $95 \%$ confidence intervals on the residual did not contain 0 . One data point (and thus 1 subject) was identified as an outlier. Further investigation revealed that the subject associated with this data point had a transient ischemic attack within a few days of the FTT assessment, and thus the relationship between the median IKI over the 28-day interval and the average tapping speed was affected by this event. As a result, we removed this subject from the analysis.

Of the 225 subjects fully enrolled in the ISAAC study (164 female, 61 male), 115 had been living alone since the beginning of the study (103 female, 12 male). Of these 115 , 13 did not receive a study computer, as they opted to use their own. While we were able to install some software unrelated to the present analysis on these 13 subjects' personal computers, the key-logging software was not installed. This left a total of 102 subjects, from whom we identified 22 who met the exclusionary criteria described above and whose data were included for the present analysis. Most of the excluded subjects were excluded because of the lack of a sufficient amount of computer login data within the window for comparison with the FTT. Since the ISAAC study is a natural history study of activity and computer use monitored unobtrusively over time in order to detect normative cognitive and motor change with aging, no attempt was made to encourage subjects to use the computer more frequently than their normal patterns dictated. While the requirement that a certain amount of login data fall within a window of an FTT examination was necessary to validate the proposed methodology, we note that in practice this is not required. In the ISAAC cohort, $40 \%$ of the subjects are daily computer users, and almost all use the computer on a weekly or monthly basis. As a result, the proposed method could be employed on most subjects in our cohort, even though the validation to the FTT could only be demonstrated on the subset of subjects meeting the exclusionary criteria. All subjects gave informed consented in accordance with the Institutional Review Board approved procedure. The mean age $( \pm S D)$ of the subject population was $83.5 \pm 4.0$ years and ranged from 73.4 to 89 years of age. The mean education level $( \pm S D)$ was $15.8 \pm 2.5$ years and ranged from 12 to 20 years of education. Twenty of the subjects were women, and 20 were right-handed according to the Handedness Inventory. All subjects were cognitively intact (no dementia or mild cognitive impairment), with a Clinical
Dementia Rating Scale (Morris, 1993) score of 0. No subjects possessed symptoms of Parkinson's disease (as clinically assessed by a combination of informant report, subject history, medical records, and observation) or stroke. Three of the subjects had been identified as having symptoms of depression within the last 2 years (also as clinically assessed by a combination of informant report, subject history, medical records, and observation). At the start of the ISAAC study, all subjects who were unable to independently compose and send e-mail or who requested computer instruction participated in a six-session training program on basic computer use. Others, who were enrolled after the study began, were provided with individual computer training. All subjects had a personal computer provided in their home as part of the ISAAC study or opted to use their own computer. All subjects had had a computer in their home for at least 2 years with the same login user name prior to the earliest computer data used in this study.

\section{The FTT and test administration}

The FTT procedure used in this study was a slight variant of that described in the introduction. Instead of asking the subject to complete five trials, we administered only three trials each for both the dominant and nondominant hands. Each trial consisted of the subject placing his or her palm flat on the manual finger tapper with the index finger of the hand under test placed on the lever. The subject was then instructed to tap as many times as possible in a 10-s interval while a clinician timed the 10 -s trial with a stopwatch. The manual finger-tapper boards used were obtained from Reitan Neuropsychology Laboratory (www.reitanlabs. com). The same clinician administered all of the tests. The FTT score is reported as the mean number of taps recorded during the three trials, for each hand. For a direct comparison of the FTT with our IKIs (defined in the next subsection), we calculated the average tapping time, $T_{\mathrm{FTT}}$, as

$T_{\mathrm{FTT}}=(10 \mathrm{~s}) / N$,

where $N$ is the number of taps recorded for the FTT.

\section{IKI collection and preprocessing}

The personal computer in each subject's home was preloaded with computer software developed by the Oregon Center for Aging and Technology (ORCATECH), which presented a study-specific login screen and logged the time stamp and the key pressed for each keyboard event during the login. The IKI was defined for each pair of keypresses as the time interval between the initial contact of the first key to the initial contact of the second key. From this set of data, we used only the assigned computer login user name 


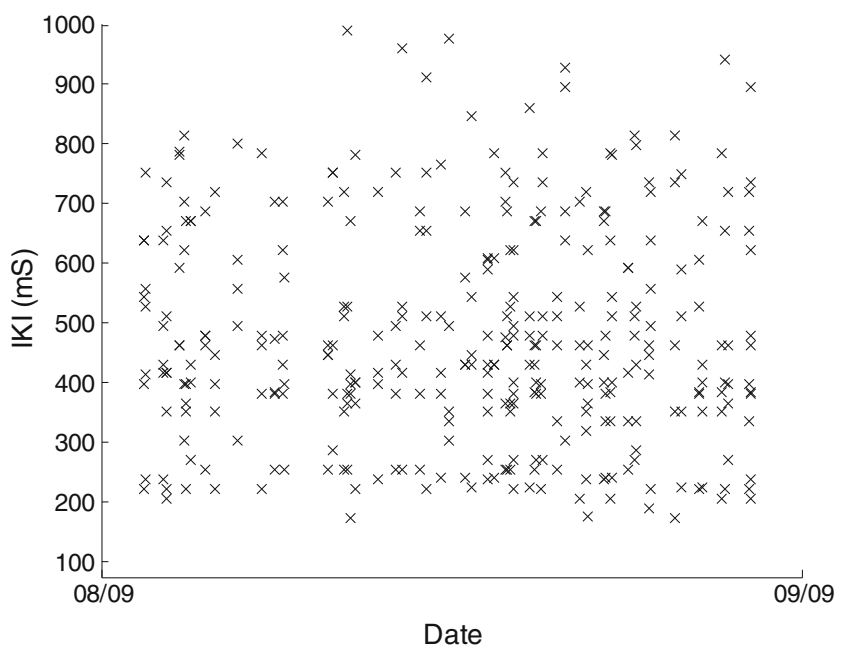

Fig. 1 User name login interkeystroke intervals (IKIs) versus date for 1 subject with highly variable times in the 28-day window centered on the associated finger-tapping (FTT) test date

characters corresponding to a successful login event that were also typed without errors (e.g., mistyped user names in which the subject needed to delete and retype some characters were not included). Figures 1 and 2 show scatterplots of the IKIs, plotted against date, for 2 of the subjects. These two figures represent the extremes of the data seen across our subject population. The data in Fig. 1 are from a subject who shows large variability in IKIs, whereas Fig. 2 shows data from a subject with much less (and more typical) variability in keystrokes. The median of all IKIs in the 28-day window centered on the corresponding FTT administration date was the measure of central tendency used for comparison with the average tapping time $\left(T_{\mathrm{FTT}}\right)$ for each subject.

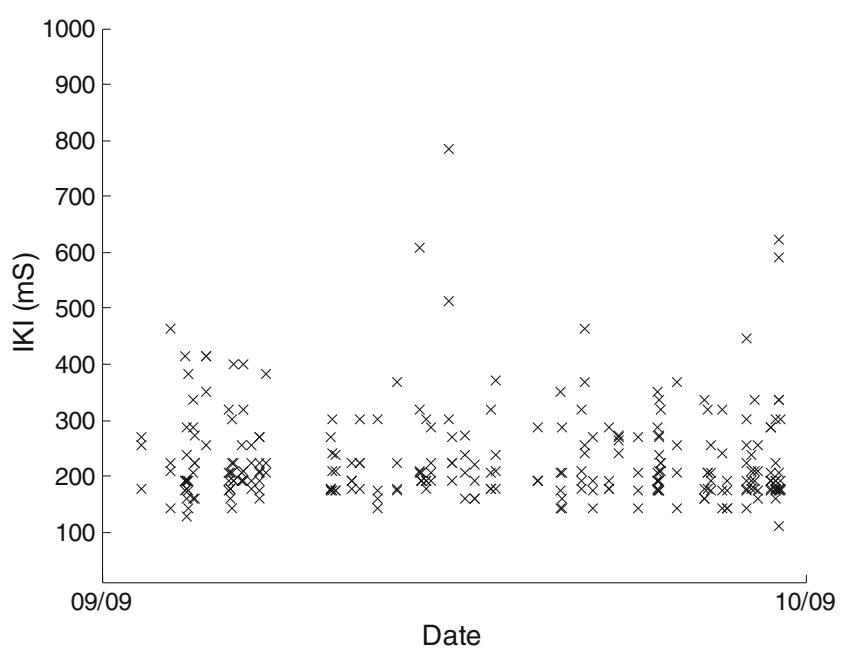

Fig. 2 User name login IKI versus date for another subject with more typical variability for the 28-day window centered on the associated FTT test date

\section{Results}

Due to the face validity of the finger flexion and extension required to generate IKI's while typing highly repeated and overlearned sequences, we hypothesized a positive correlation between the typing speed and average tapping time across subjects. To test this hypothesis, we regressed the $T_{\text {FTT }}$ times for both the dominant and nondominant hands (separately) onto the median IKI and calculated the Pearson correlation coefficient $r$. To assess the significance of the results, we tested the one-sided hypothesis that the correlation is positive versus the null hypothesis that the correlation is not positive, using Student's $t$ tests separately for both the dominant and nondominant hands. To further quantify the value of the correlation coefficient, we calculated confidence intervals using the Fisher $z$-transform method. All data analysis was done using MATLAB Version 7.6.0 (R2008a; The Mathworks, Inc.).

Table 1 shows population means and standard deviations $\left(S D\right.$ s) for both FTT scores and mean tapping speeds $T_{\text {FTT }}$ (dominant and nondominant hands), as well as median IKI times. The regression line from the median IKI to the nondominant- and dominant-hand $T_{\text {FTT }}$ values are shown in Figs. 3 and 4, respectively. The correlations, $p$ values from the hypothesis tests, and confidence intervals are shown in Table 2. The calculated correlation coefficient values of .77 and .70 between the nondominant and dominant hands, respectively, show that much of the variability in $T_{\text {FTT }}$ is explained by the median IKI. In both cases, the hypothesis tests showed that the positive correlation was significant at the $5 \%$ level $(p s<.0001$ and .0002 for the nondominant and dominant hands, respectively). For further comparisons, we calculated the sample correlation between dominant- and nondominant-hand FTTs in all 225 subjects of the ISAAC cohort and tested significance at the $5 \%$ level. This correlation was $.75(p<.0001)$, which is close to the correlations between $T_{\text {FTT }}$ and median IKIs for both hands. In the literature, the test-retest reliability of the FTT has been reported to be between .58 and .93 (Strauss et al., 2006) over a variety of studies, conducted among both patient and normal populations. This places the median IKI-to- $T_{\mathrm{FTT}}$

Table 1 Population means and standard deviations ( $S D$ s) for FTT scores and mean tapping speed $T_{\mathrm{FTT}}$ (for both the dominant and nondominant hands), as well as median IKI times

\begin{tabular}{lll}
\hline & Mean & $S D$ \\
\hline FTT dominant hand (score) & 39.7 & 8.2 \\
FTT nondominant hand (score) & 35.8 & 8.2 \\
$T_{\text {FTT dominant hand (ms) }}$ & 264 & 61 \\
$T_{\text {FTT nondominant hand (ms) }}$ & 295.1 & 73.8 \\
Median IKI (ms) & 356.2 & 155.2 \\
\hline
\end{tabular}




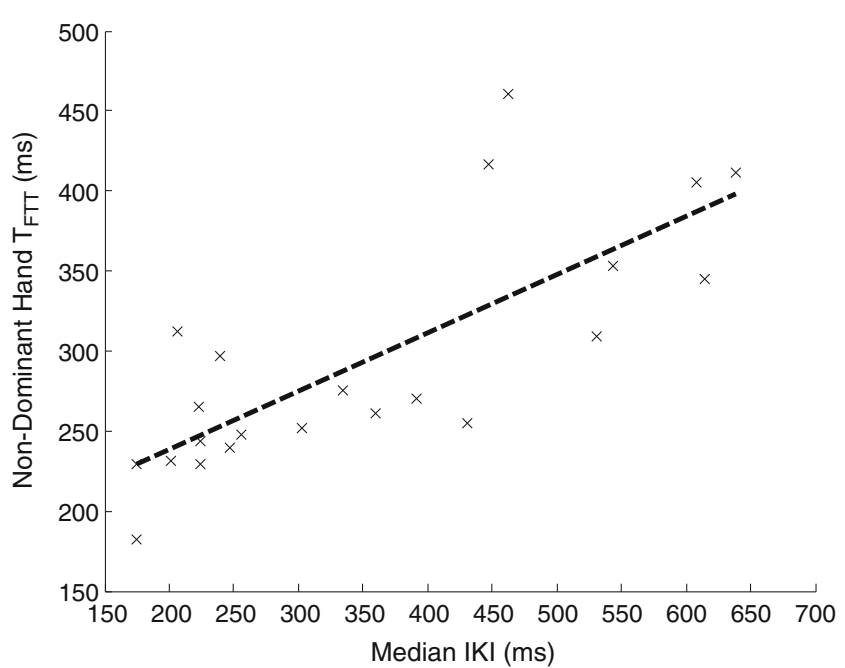

Fig. 3 Regression line (black dashed line) for the predictor variable, median IKI, to the nondominant-hand average tapping speed $\left(T_{\mathrm{FTT}}\right)$, calculated from the FTTs for 22 subjects, along with individual measurement pairs (black x's) for each subject

correlations firmly within the test-retest range of the FTT for both the nondominant and dominant hands.

\section{Discussion}

In this study, we proposed the median interkeystroke intervals of memorized and frequently used computer login user names as a measure of simple motor speed and compared that measure as a surrogate for the average tapping speed derived from the FTT score, with initial validation in an elderly subject population living independently. The results of the correlation analysis were

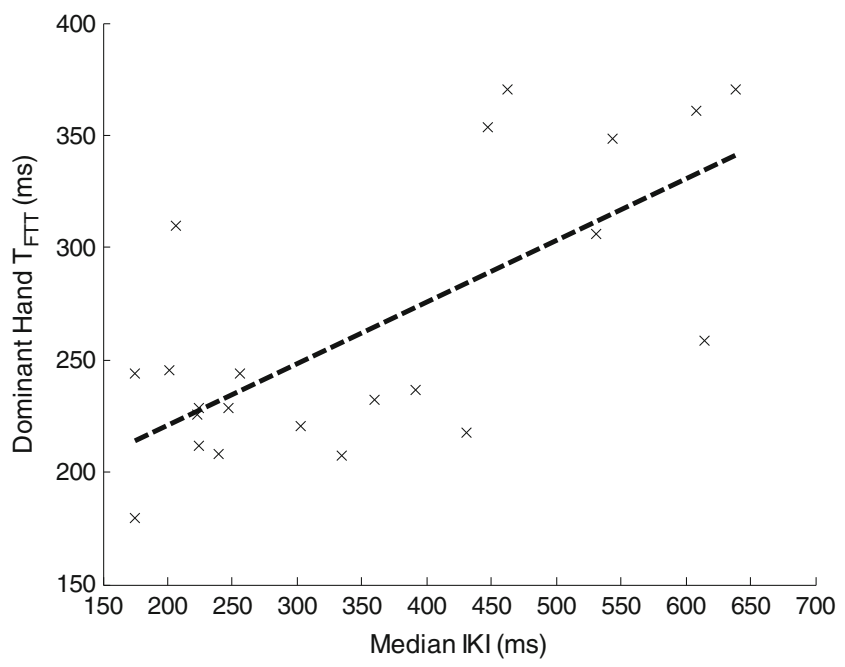

Fig. 4 Regression line (black dashed line) for the predictor variable, median IKI, to the dominant-hand average tapping speed ( $\left.T_{\mathrm{FTT}}\right)$ for 22 subjects, along with individual measurement pairs (black x's) for each subject
Table 2 Correlation coefficient estimates between median IKI time and $T_{\mathrm{FTT}}$ for dominant and nondominant hands, $p$ values for hypothesis tests of significance of positive correlations, and $95 \%$ confidence intervals of the correlation estimates

\begin{tabular}{llll}
\hline & $r$ & $p$ Value & $95 \%$ Confidence Intervals \\
\hline Nondominant hand & .77 & $<.0001$ & $(.516, .90)$ \\
Dominant hand & .70 & $<.0002$ & $(.395, .866)$ \\
\hline
\end{tabular}

significant and show that the proposed method accounts for much of the variability in the FTT across subjects. Furthermore, the fact that the correlation is within the range of the FTT's test-retest reliability for both the nondominant and dominant hands, combined with the face validity of the typing motion, suggests that the proposed method has capabilities similar to the FTT for assessing simple motor speed.

The reason we selected the median as a suitable measure of central tendency - instead of the mean, which is a more intuitive choice for comparison with mean tapping speedis because of the large variability in the IKI distributions of some subjects (such as the one whose data are shown in Fig. 1). The median is much more robust to this wide spread of data than is the mean, and it was this property of robustness that we desired in an estimator of central tendency for these highly variable data. Furthermore, since the support of the distribution underlying these data is the positive real line, there is a natural skewness associated with the IKI distributions. Specifically, things such as pauses between letters not associated with the motor aspect of typing (i.e., if someone sneezes or hears the phone ring during the login sequence) will show up as slower IKIs and cause outliers only on one side of the distribution. The effect of these types of events is what we sought to minimize with the choice of median IKI as the measure of motor speed.

To understand the difference in correlations between the median IKIs and the dominant- and nondominant-hand values of $T_{\mathrm{FTT}}$, we considered the keyboard placement of the most commonly used letters that appeared in the user name character strings of the 22 subjects. Due to the wellknown property of the QWERTY keyboard, where the most common letters appear on the left side, any touch typist would use their left hand more frequently than their right. While our subjects are not all trained typists, it is reasonable to assume that many of the characters closer to the left hand would be pressed by the left hand even in nonskilled typing styles. Since 20 of our 22 subjects were right-handed, more of the data used in our study were generated by the nondominant hand, which might account for the better correlation between the median IKI and the nondominant hand. 
Although these results, along with the similarity of typing with tapping, show both the accuracy and the face validity of the proposed method as a possible early screening tool or surrogate for the FTT, the main advantage of this technique is its ability to assess behavioral measures unobtrusively and frequently. In particular, the IKI data can be collected unobtrusively in a subject or patient's residence on a frequent basis, without the need for a trained assessor to administer testing. In contrast to FTTs, this type of assessment is not subject to interrater variability. As a result, within-subjects trends in motor speed may allow us to detect problems much earlier than traditional psychomotor tests. Changes in motor speed can be detected when they occur - either in real time, as part of a clinical alerting system, or retrospectively, through offline data analysis and interpretation. Furthermore, intraindividual variability in motor function, manifested as variability in IKIs, may itself be an important indicator or predictor of function (Bielak, Hultsch, Strauss, Macdonald, \& Hunter, 2010). This is something that is not currently collected as part of the FTT and cannot be evaluated until many FTTs have been administered over many years. Thus, the proposed method would allow for both short- and long-term variability to also be assessed. This issue of periodic and infrequent assessments has been discussed in the context of dementia prevention trials (Kaye, 2008) for assessment methods in general, and addressing these issues in commonly administered tests (such as the FTT) with in-home monitoring may introduce new analysis tools and diagnostic aids for both researchers and clinicians.

Despite the promise of the proposed method and the validity demonstrated in the ISAAC cohort, there are some limitations. First, some of the uses of the FTT are based on assessing differences in performance between hands, such as when detecting the presence of brain lesions (Strauss et al., 2006). The currently proposed method does not distinguish between the hands (or fingers), since we use the same predictor for both the dominant and nondominant values of $T_{\mathrm{FTT}}$. Second, focusing only on the sequential depression of the same set of computer keys (a user name string) greatly reduces the use of the wide variety of key capture data that can be obtained over the course of monitoring computer sessions over time. Additionally, the validation of the proposed methodology was conducted with healthy elderly subjects who live independently. While these subjects are potentially at high risk for adverse outcomes, generalization of this technique to other patient populations across a wider age and demographic profile will require further studies. Another apparent shortcoming of the proposed technique is the fact that unobtrusive measurements are subject to significantly higher variability than are those performed in controlled environments. The results of the analysis presented in this article suggest that this variability is well compensated for by the very large number of samples. It is also possible to speculate that the variability of the raw IKI data themselves could eventually be used for additional assessment of the cognitive as well as motor functionality of continuously monitored individuals (Bielak et al., 2010).

\section{Conclusion}

In this article, we proposed a new method for assessing motor speed, based on computer interkeystroke intervals of highly learned and frequently repeated sequences, and we demonstrated that the data collected using this method correlate well with the average tapping speeds derived from the FTT. The resulting significant correlation across subjects suggests that the IKI measure is a useful alternative to the conventional FTT, overcoming some of the limitations in its current use. Moreover, the IKI-based technique may enable nearly continuous assessment and thereby be used for early detection of changes in motor and related functions. Since the IKI during regular typing can incorporate cognitive aspects of function, the IKI-based approach may also provide sensitive, unobtrusive measures of cognitive function.

Author note The authors thank the volunteer subjects who participated in this research and the staff from the Oregon Center for Aging and Technology who assisted in the study. This work was supported in part by National Institutes of Health Grants P30AG008017, P30-AG024978, R01-AG024059, and K01-AG23014; Department of Veterans Affairs Grants P30-AG008017 and M01RR000334; and the Intel Corporation. T.H. has a significant financial interest in Intel Corporation, a company that provided funds to purchase some of the computers used in this study. Intel Corporation may have a commercial interest in the results of this research. This potential conflict has been reviewed and managed by Oregon Health and Science University.

\section{References}

Bielak, A. A., Hultsch, D. F., Strauss, E., Macdonald, S. W., \& Hunter, M. A. (2010). Intraindividual variability in reaction time predicts cognitive outcomes 5 years later. Neuropsychology, 24, 731-741.

Buracchio, T., Dodge, H. H., Howieson, D., Wasserman, D., \& Kaye, J. (2010). The trajectory of gait speed preceding mild cognitive impairment. Archives of Neurology, 67, 980-986.

Camicioli, R., Howieson, D., Oken, B., Sexton, G., \& Kaye, J. (1998). Motor slowing precedes cognitive impairment in the oldest old. Neurology, 50, 1496-1498.

Christianson, M. K., \& Leathem, J. M. (2004). Development and standardisation of the Computerised Finger Tapping Test: Comparison with other finger tapping instruments. New Zealand Journal of Psychology, 33, 44-49.

Gentner, D. R., Larochelle, S., \& Grudin, J. (1988). Lexical, sublexical, and peripheral effects in skilled typewriting. Cognitive Psychology, 20, 524-548. 
Godefroy, O., Roussel, M., Despretz, P., Quaglino, V., \& Boucart, M. (2010). Age-related slowing: Perceptuomotor, decision, or attention decline? Experimental Aging Research, 36, 169189.

Godefroy, O., Spagnolo, S., Roussel, M., \& Boucart, M. (2010). Stroke and action slowing: Mechanisms, determinants and prognosis value. Cerebrovascular Diseases, 29, 508-514.

Jiménez-Jiménez, F. J., Calleja, M., Alonso-Navarro, H., Rubio, L., Navacerrada, F., Pilo-de-la-Fuente, B., et al. (2011). Influence of age and gender in motor performance in healthy subjects. Journal of the Neurological Sciences, 302(1-2), 72-80.

Jimenez-Jimenez, F. J., Rubio, L., Alonso-Navarro, H., Calleja, M., Pilo-de-la-Fuente, B., Plaza-Nieto, J. F., et al. (2010). Impairment of rapid repetitive finger movements and visual reaction time in patients with essential tremor. European Journal of Neurology, $17,152-159$.

Kaye, J. (2008). Home-based technologies: A new paradigm for conducting dementia prevention trials. Alzheimer's \& Dementia, 4(1, Suppl. 1), S60-S66.

Kaye, J., Maxwell, S. A., Mattek, N., Hayes, T., Dodge, H., Pavel, M., et al. (in press). Intelligent systems for assessing aging changes: Home-based, unobtrusive and continuous assessment of aging. Journals of Gerontology.

Kaye, J., Oken, B. S., Howieson, D. B., Howieson, J., Holm, L. A., \& Dennison, K. (1994). Neurologic evaluation of the optimally healthy oldest old. Archives of Neurology, 51, 12051211.
Kiziltan, E., Barut, C., \& Gelir, E. (2006). A high-precision, low cost system for evaluating finger-tapping tasks. International Journal of Neuroscience, 116, 1471-1480.

Levey, A., Lah, J., Goldstein, F., Steenland, K., \& Bliwise, D. (2006). Mild cognitive impairment: An opportunity to identify patients at high risk for progression to Alzheimer's disease. Clinical Therapeutics, 28, 991-1001.

Morris, J. C. (1993). The Clinical Dementia Rating (CDR): Current version and scoring rules. Neurology, 43, 2412-2414.

Morrison, M. W., Gregory, R. J., \& Paul, J. J. (1979). Reliability of the Finger Tapping Test and a note on sex differences. Perceptual and Motor Skills, 48, 139-142.

Ott, B. R., Ellias, S. A., \& Lannon, M. C. (1995). Quantitative assessment of movement in Alzheimer's disease. Journal of Geriatric Psychiatry and Neurology, 8, 71-75.

Shaffer, L. H. (Ed.). (1973). Latency mechanisms in transcription. New York: Academic Press.

Shigematsu, R., Rantanen, T., Saari, P., Sakari-Rantala, R., Kauppinen, M., Sipila, S., et al. (2006). Motor speed and lower extremity strength as predictors of fall-related bone fractures in elderly individuals. Aging Clinical and Experimental Research, 18, 320-324.

Strauss, E., Sherman, E. M. S., \& Spreen, O. (2006). A compendium of neuropsychological tests: Administration, norms, and commentary (3rd ed.). Oxford: Oxford University Press.

Wefel, J. S., Hoyt, B. D., \& Massma, P. J. (1999). Neuropsychological functioning in depressed versus nondepressed participants with Alzheimer's disease. Clinical Neuropsychology, 13, 249-257. 